\title{
Energy calibration via correlation using an adaptive mesh refinement
}

\author{
Daniel Maier*, Olivier Limousin*, Geoffrey Daniel ${ }^{* \dagger}$ \\ *AIM, CEA, CNRS, Université Paris-Saclay - Université Paris Diderot \\ Sorbonne Paris Cité - F-91191 Gif-sur-Yvette Cedex, France - Email: daniel.maier@cea.fr \\ †École des Ponts ParisTech - 77420 Champs-sur-Marne, France
}

\begin{abstract}
Energy calibration via correlation has proven to be a powerful calibration method especially in the case of calibration data sets with low counting statistics. This work reports on an evolved version of this approach that uses an adaptive mesh refinement to sample the parameter space which results in the same calibration performance but in a considerably reduced computation time.
\end{abstract}

\section{INTRODUCTION}

A precise energy calibration is a critical step for many experiments using X-ray and gamma-ray detectors, like spectrometry in general, X-ray fluorescence analysis, Compton imaging, source identification... Non-linear effects introduced by the detector response as well as non-linear effects within the signal processing chain, like in amplifier ASICs or ADCs, often require to use non-linear models for the energy calibration, i.e. a parameter space with a dimensionality bigger than two. In addition, repetitive re-calibration that is used to correct time-dependent response instabilities, or a strong pixelation of the detector result in low counting statistics per detector channel implying a reduced accuracy of the energy calibration. Section II shortly summarizes the main idea behind the classical energy calibration via correlation (ECC) approach. Section III presents an evolved parameter sampling and optimization method used for ECC, the adaptive mesh refinement (AMR). Section IV gives an outlook for the implementation of an adaptive reduction of dimensionality, a feature foreseen for the next version of ECC. Section V summarizes the results.

\section{A FIRST SOLUTION: ECC}

A method that uses an Energy Calibration via Correlation approach was presented [1] to address the problems of low counting statistics in the calibration data set. The idea of ECC is not to use a peak fitting approach, which examines only specific line positions, but to use the complete spectral response of the calibration measurement. The procedure of ECC can be summarized as follows:

1) Make a synthetic line spectrum based on prior knowledge of the used calibration source (Fig. 1 (a)).

2) Modify the line spectrum to a synthetic energy spectrum by applying the detector response model. This model corrects for a non-ideal detector response and includes typically the energy resolution and the quantum efficiency of the detector (Fig. 1 (b)).

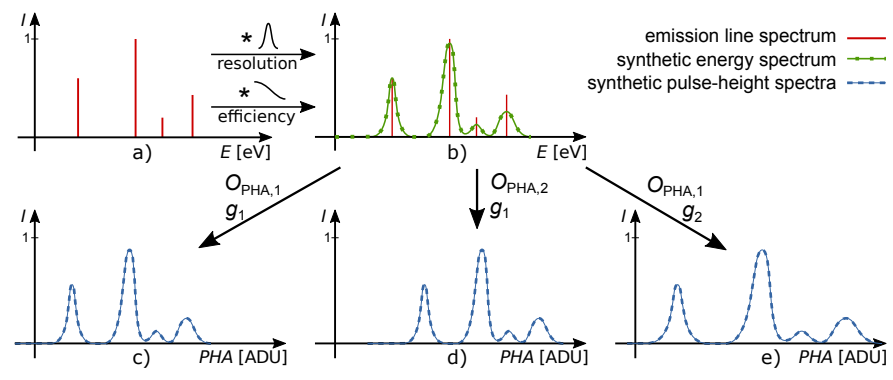

Fig. 1. Concept of ECC: creating synthetic energy spectra (a-b) and transforming them into synthetic pulse-height spectra (c-e) that are correlated with the observed pulse-height spectrum.

3) Discretize the parameter space and calculate for every combination of parameters the corresponding synthetic pulse-height spectrum (Fig. 1 (c) to (e)).

4) Compute the correlation value of all synthetic pulseheight spectra with the observed pulse-height spectrum.

5) The best calibration parameters are found by searching for the highest correlation (Fig. 2).

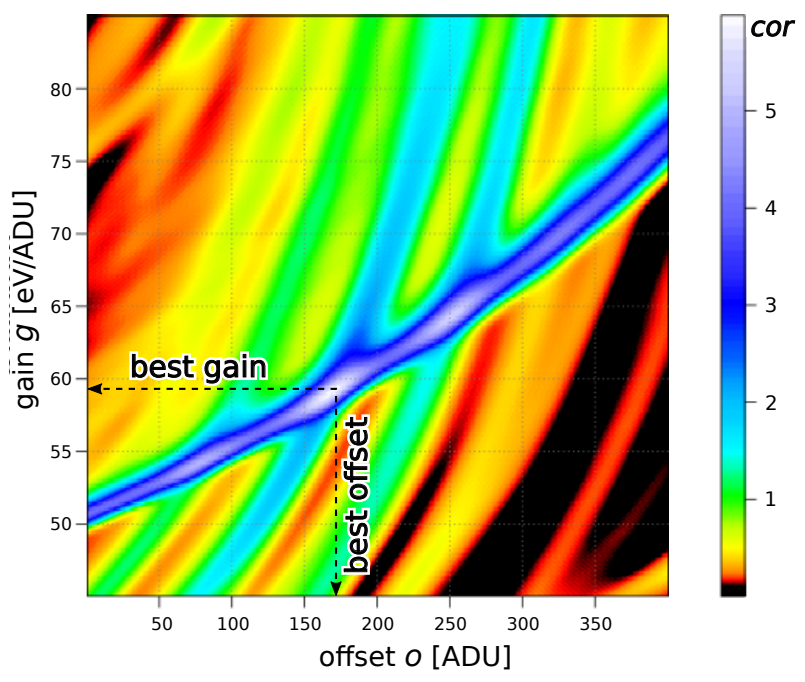

Fig. 2. Correlation map: the correlation value cor indicates the goodness of the parameter set which is here defined by the offset $o$ and the gain $g$.

The correlation value is defined as

$$
\text { cor }=\sum_{i=0}^{N-1} S_{\mathrm{syn}, i} \cdot S_{\mathrm{obs}, i}
$$


where $i$ is the binning index of the synthetic pulse-height spectrum $S_{\text {syn }}$ and the observed pulse-height spectrum $S_{\text {obs }}$ and $N$ is the total number of bins. The higher the correlation value the better is the match between the two spectra. Therefore, the correlation value can be used as a measure of the goodness of the parameter set.

Even though the presented examples show a linear calibration, the concept of ECC is not limited to any number of parameters. An example of a non-linear ECC using three parameters can be found in [1].

Compared to a peak fitting approach that is based on finding PHA-energy pairs and fitting these pairs to the proposed calibration model, ECC performs considerably better, especially for calibration sets with a small number of photons. Another benefit of ECC is that it is not restricted to line peaks but can use the full spectral response like Comptonization structures. See reference [1] for more details on ECC and a quantitative comparison between ECC and a Gaussian peak fitting approach.

\section{ADAPTIVE MESH REFINEMENT}

The equally spaced homogeneous grid that was used for the previous presented implementation of ECC samples each region of the parameter space equally fine. If a high parameter resolution is required and the parameter space is large because of high dimensionality or large pixel-to-pixel variations, the computation time for the calibration becomes an issue. This is especially true for applications which aim for near realtime calibration or re-calibration or applications which have many detection channels, i.e. strong pixelation. Adaptive mesh refinement is a numerical analysis method that samples the parameter space dynamically during the computation time with an increasing resolution for interesting regions. It is frequently used in simulations of turbulent regions of fluid dynamics [2].

\section{A. Adaptive mesh zooming}

Compared to the previous, classical ECC procedure described in Sect. II, only the $3^{\text {rd }}$ step is changing for $\mathrm{ECC}_{\mathrm{AMR}}$. The adaptive mesh approach samples the parameter space in two steps. First, an initial homogeneous mesh, similar to the one used in the classical approach but with a much lower parameter resolution, is applied and the corresponding correlation map is calculated. Based on the obtained correlation values $\operatorname{cor}_{0, j}$, regions are classified as interesting if

$$
\begin{aligned}
\operatorname{cor}_{0, j} & >t_{0} \quad \text { for all mesh elements } j \\
t_{0} & =f_{0} \cdot \operatorname{cor}_{0}^{*}
\end{aligned}
$$

where $f_{0}$ defines a fraction of the best obtained initial correlation value $\operatorname{cor}_{0}^{*}$. The initial zooming threshold $t_{0}$ triggers a zoom into an interesting region with a three times increased resolution for each parameter; i.e. for two parameters an interesting region is sampled with a sub-resolution of $3 \times 3$. The correlation values of each of these 9 sub-regions are calculated and new interesting regions are defined via

$$
\begin{aligned}
\operatorname{cor}_{i, j} & >t_{i} \\
t_{i} & =t_{i-1}+f \cdot\left(\operatorname{cor}_{i}^{*}-t_{i-1}\right) \quad \text { for } i=1 . . N_{\mathrm{z}}
\end{aligned}
$$

where $i$ is the iteration index and $N_{\mathrm{z}}$ defines the maximal number of iterative zoomings. The zooming-threshold definition via two independent parameters $f_{0}$ and $f$ is motivated by the idea to have a less strict selection for the first zooming but an increasing harder selection for the following iterations. Figure 3 shows the described zooming method schematically.

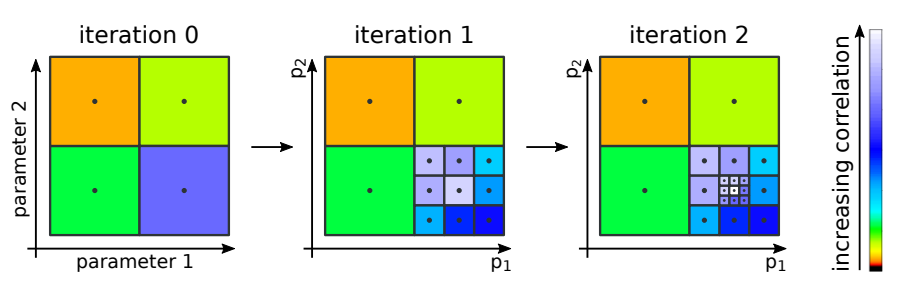

Fig. 3. Schematic zooming into interesting regions. The parameter set for each region is indicated with a dot. For iteration 2 the total number of calculated correlations is $4+8+8=20$.

\section{B. Adaptive mesh expansion}

Because the center of a parameter region is used to calculate the correlation value for this region, the region might appear as not interesting even if the optimal parameter set is positioned within the region but close to the edge of the region. In this case, the rough sampling prevents from finding the optimal parameter set.

To minimize this problem, a mesh expansion method is developed that allows to step backwards iteratively and zoom into a region that was initially classified as uninteresting if the difference of the iteration index $i$ between neighboring regions is bigger than two. For an element with index $i_{e}$ and its neighboring elements with index $i_{e, k}$, the expansion condition becomes

$$
\left|i_{e}-i_{e, k}\right|>2 \text { for all } e \text { and } k .
$$

In order to keep the number of neighbors low, only neighbors to the edges of a region are considered. This is especially important for a high dimensional parameter space. Given the dimensionality of the parameter space as $d$, the number of edge neighbors that differ only in one parameter value is $2 \cdot d$ while it is maximally $3^{d}-1$ for all possible neighbors.

After the expansion, the zooming criterion is applied for the new region according to the saved thresholds $t_{i}$. Finally the expansion condition is checked for the new region. Due to this method, the algorithm expands into interesting parameter regions even if they seemed to be uninteresting within the previous iterations. In this way, the expansion method works as a local gradient ascent optimizer.

Figure 4 shows schematically the described expansion method followed by a re-zoom. After the zooming iteration $i=2$ ends, the zooming-threshold $t_{2}$ is calculated. The region indicated in red exceeds this threshold and a zoom will be conducted for $i=3$. This would violate the condition of Eq. (6) so that an expansion is conducted before iteration 3 begins. After the neighboring region is expanded to $i=1$, it is re-zoomed according to threshold $t_{1}$. Finally, the expanding condition is checked again and another expansion might occur. If no more expansion is to be done, iteration $i=3$ starts. 


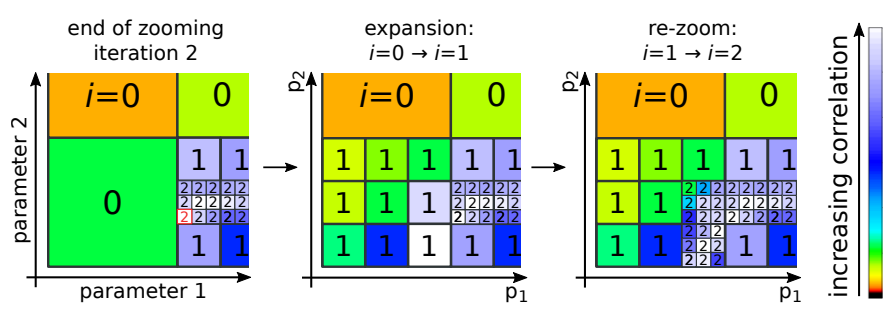

Fig. 4. Schematic expansion into an interesting region followed by a re-zoom. After the re-zoom, another expansion to the bottom region (not shown) might occur or the next iteration $i=3$ starts.

\section{Calibration example}

Figure 5 shows a correlation map for the calibration of a camera system [3] that is based on a CdTe Caliste-HD detector [4]. The calibration uses an Am-241 source and calculates the best calibration parameters via classical ECC which was conducted by using $\mathrm{ECC}_{\mathrm{AMR}}$ with no iteration $(i=0)$ and with a fine resolved initial mesh of $\Delta o=5 \mathrm{ADU}$ and $\Delta g=0.25 \mathrm{ADU} / \mathrm{keV}$. The parameter space is equally sampled with $1000 \times 720$ sampling points and the calculation of the $7.2 \cdot 10^{5}$ correlations takes approximately 100 seconds on an ordinary computer with single core use.

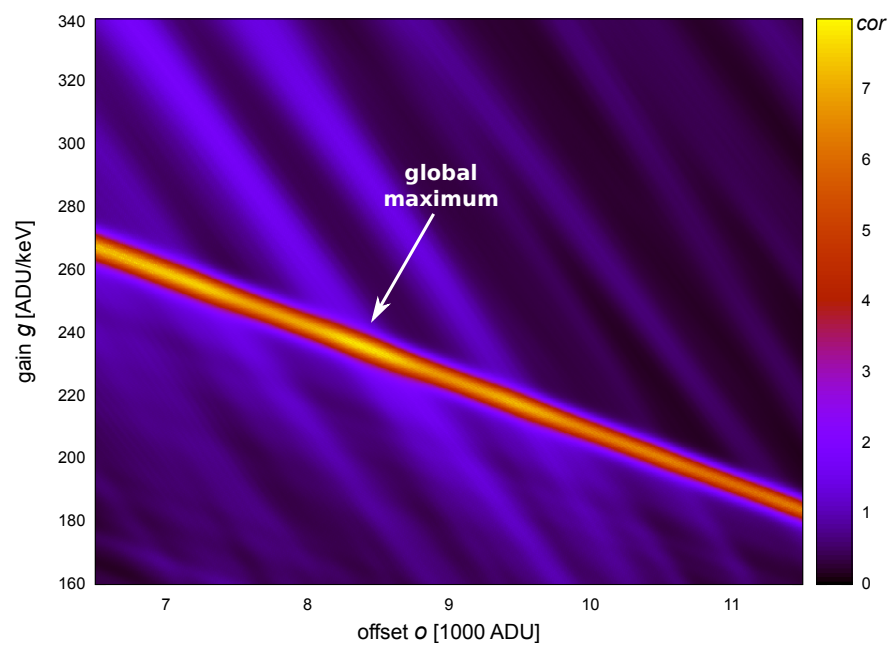

Fig. 5. Correlation map for an Am-241 calibration obtained via a classical fine mesh ECC with a resolution of $\Delta o=5 \mathrm{ADU}$ and $\Delta g=0.25 \mathrm{ADU} / \mathrm{keV}$.

This realistic data set shows that the optimization problem is not convex but has multiple local maxima. Furthermore, due to absorption of low energy photons in the camera housing $(0.5 \mathrm{~mm} \mathrm{Al})$, the source spectrum is dominated by the emission line around $60 \mathrm{keV}$. Consequently, all parameter combinations that match the synthetic $60 \mathrm{keV}$ line with the observed line result in a large correlation which can be seen by the yellow line in Fig. 5. Nevertheless, the obtained parameter set for the best correlation fits the measurement well, see Fig. 6 .

Figure 7 shows for the same calibration measurement the result of $\mathrm{ECC}_{\mathrm{AMR}}$ after 5 iterations. The initial grid was chosen to $\Delta o=500 \mathrm{ADU}$ and $\Delta g=10 \mathrm{ADU} / \mathrm{keV}$, leading to 400 initial correlations and a final resolution of $\Delta o \approx 2 \mathrm{ADU}$

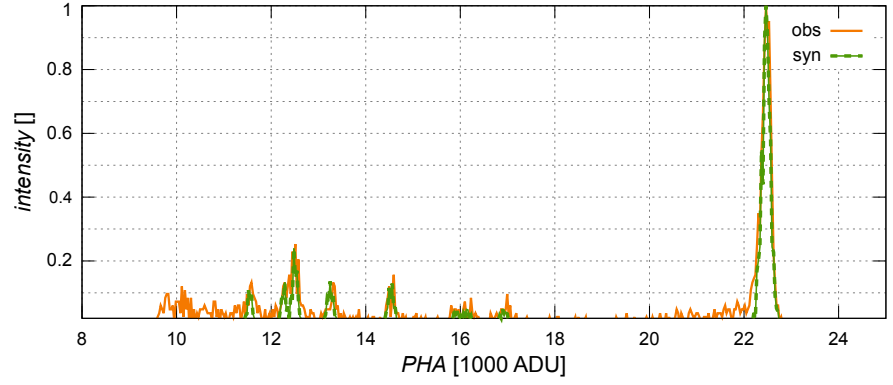

Fig. 6. Observed (obs) and best synthetic (syn) spectra for an Am-241 calibration for a single detector channel. The observation has 2590 events. Both spectra are normalized to a maximal intensity of 1 .

and $\Delta g \approx 0.05 \mathrm{ADU} / \mathrm{keV}$. The threshold parameters were set to $f_{0}=0.6$ and $f=0.7$. The numbers of computed correlations for each iteration are shown in Fig. 8. In total, 1602 correlations are calculated which is 450 times less compared to classical ECC. The number of correlations directly measures the computation time within ECC. The total computation time is about 2 seconds, which is, compared to classical ECC, a reduction by a factor of 50 , including also reading the input data, binning the spectra, and writing the results.

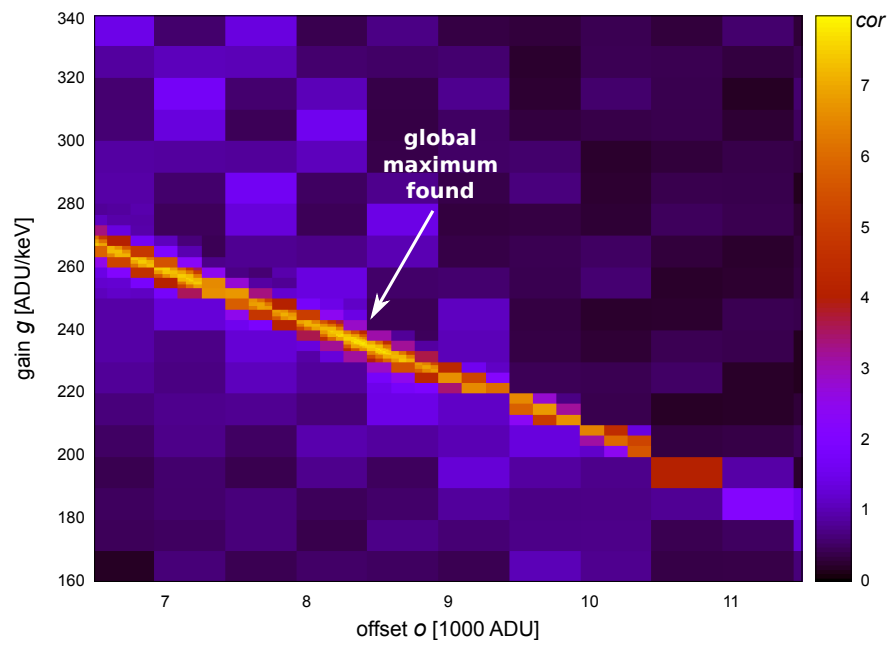

Fig. 7. $\mathrm{ECC}_{\mathrm{AMR}}$ correlation map after 5 iterations. The final resolution at the global maximum is $\Delta o \approx 2 \mathrm{ADU}$ and $\Delta g \approx 0.05 \mathrm{ADU} / \mathrm{keV}$.

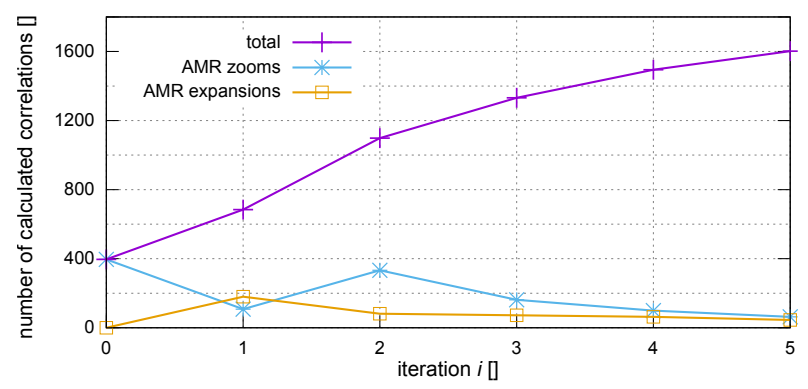

Fig. 8. Number of calculated correlations as a function of the iteration $i$. The total number is the cumulative sum of the number of zooms and the number of expansions. In total, 1602 correlations are calculated. 
Figure 9 shows the correlation map for the same dataset but using $\mathrm{ECC}_{\mathrm{AMR}}$ with disabled adaptive mesh expansion. The global maximum cannot be found in this case because the initial grid shows near the global maximum a relatively low correlation value of $c o r \approx 3.2$ which is only $44 \%$ of the maximal initial correlation value. A finer initial grid or a lower threshold factor $f_{0}$ could resolve this problem but only at the expense of an increased computation time.

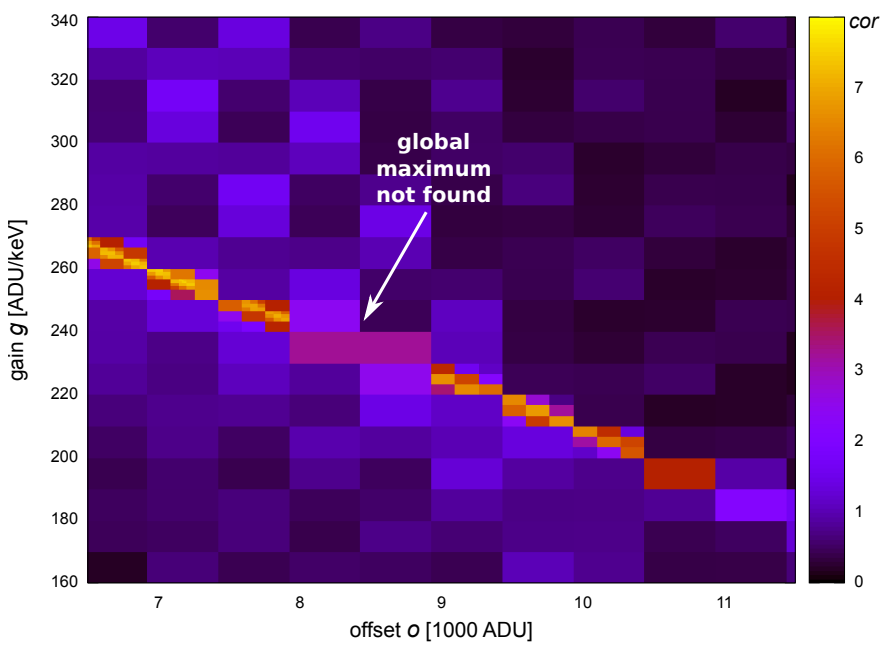

Fig. 9. ECC $_{\mathrm{AMR}}$ correlation map after 5 iterations with disabled adaptive mesh expansion. The global maximum is not found.

\section{OUTLOOK}

In the current version, the resolution of each parameter is increased by a factor of 3 for each iteration. If the required resolutions of the parameters are considerably different, it would be beneficial if the zooming factor could change during the computation for each parameter individually. In particular, an implementation is foreseen where the zooming of a parameter stops if a given resolution is obtained. This would result in an adaptive reduction of the dimensionality of the parameter space - a consistent further development in the sense of an adaptive mesh. Figure 10 shows this idea schematically for the case of two parameters. The stopping criterion, which is in the current version defined by the number of iterations, would be reached if all required parameter resolutions are obtained.
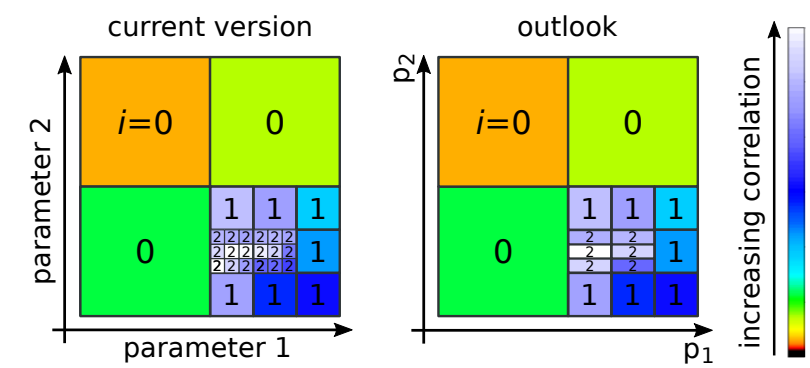

Fig. 10. After iteration $i=1$, the zooming is reduced from $3 \times 3$ (current version) to $1 \times 3$ (next version), i.e. only 3 and not 9 zooms are calculated for each interesting region. The stopping condition is reached when the resolution requirements of all parameters are achieved and no more zooming is required.

\section{CONCLUSIONS}

ECC is compatible with an adaptive mesh refinement that reduces the computation time dramatically: in the given example, the computation time is reduced by a factor of $\sim 450$ for an even slightly better parameter resolution compared to the classical ECC approach. Both methods, classical $\mathrm{ECC}$ and $\mathrm{ECC}_{\mathrm{AMR}}$, result in a maximal calibration error of $\Delta E=275 \mathrm{eV}$ for the shown example of an Am-241 calibration.

The application of AMR requires to use an adaptive expansion method in order to explore regions that are interesting but that seemed to be uninteresting during previous iterations. The expansion works recursively at the end of each iteration, so that the algorithm always looks for the best correlation value for a given parameter resolution.

Even though the algorithm is shown for only two parameters and for the example of energy calibration, it can be applied for any multi-dimensional optimization problem. Like ECC, $\mathrm{ECC}_{\mathrm{AMR}}$ is highly parallelizable with a total memory usage of less than $100 \mathrm{MB}$ for the given examples.

\section{REFERENCES}

[1] D. Maier, O. Limousin, Energy calibration via correlation, Nuclear Instruments and Methods in Physics Research A 812 (2016) 43-49. arXiv:1512.04584, doi:10.1016/j.nima.2015.11.149.

[2] M. Berger, P. Colella, Local adaptive mesh refinement for shock hydrodynamics, Journal of Computational Physics 82 (1) (1989) 64 - 84. doi:https://doi.org/10.1016/0021-9991(89)90035-1.

[3] D. Maier, C. Blondel, C. Delisle, O. Limousin, J. Martignac, A. Meuris, F. Visticot, G. Daniel, P.-A. Bausson, O. Gevin, G. Amoyal, F. Carrel, V. Schoepff, C. Mahé, F. Soufflet, M.-C. Vassal, Second generation of portable gamma camera based on Caliste CdTe hybrid technology, Nuclear Instruments and Methods in Physics Research Section A: Accelerators, Spectrometers, Detectors and Associated Equipment 912 (2018) 338 - 342, new Developments In Photodetection 2017. doi:https://doi.org/10.1016/j.nima.2017.12.027.

[4] A. Meuris, O. Limousin, O. Gevin, F. Lugiez, I. L. Mer, F. Pinsard, M. Donati, C. Blondel, A. Michalowska, E. Delagnes, M.-C. Vassal, F. Soufflet, Caliste HD: A new fine pitch $\mathrm{Cd}(\mathrm{Zn}) \mathrm{Te}$ imaging spectrometer from $2 \mathrm{keV}$ up to $1 \mathrm{MeV}, 2011$ IEEE Nuclear Science Symposium Conference Record (2011) 4485-4488. 